\title{
Summary of the RHIC Retreat 2008
}

\author{
F. Pilat, M. Brennan, K. Brown, W. Fischer, C. Montag
}

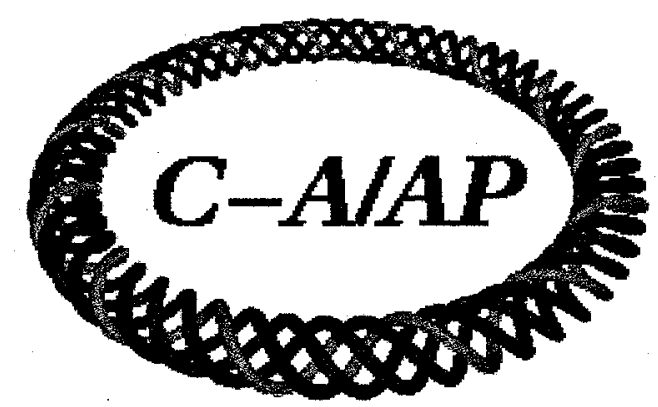

\section{Collider-Accelerator Department Brookhaven National Laboratory Upton, NY 11973}

Notice: This document has been authorized by employees of Brookhaven Science Associates, LLC under Contract No. DE-AC02-98CH10886 with the U.S. Department of Energy. The United States Government retains a nonexclusive, paid-up, irrevocable, world-wide license to publish or reproduce the published form of this document, or allow others to do so, for United States Government purposes. 


\title{
C-AD Note 318
}

\section{Summary of the RHIC Retreat 2008}

\author{
F.Pilat, M. Brennan, K. Brown, W. Fischer, C. Montag \\ July 18,2008
}

1. Introduction

2. Overview of Run- 8 and future plans

3. Preparation for gold-gold operations in Run-9

4. Preparation for polarized proton operations in Run-9

5. Machine Operations and Systems

6. The Panel Discussion

7. Plans for the shutdown

\section{Introduction}

The overall agenda, list of speakers and copy of all Retreat presentations can be found at: http://www.c-ad.bnl.gov/RHIC/retreat2008

\section{Overview of Run-8 and future plans}

The main goal of the RHIC Retreat is to review last run's performance and prepare for the next. As always though we also discussed the longer term goals and plans for the facility to put the work in perspective and in the right priority.

A straw-man plan for the facility was prepared for the DOE that assumes 30 cryoweek and running 2 species per year. The plan outlines RHIC operations for 2008-2012 and integrates well accelerator and detector upgrades to optimize the physics output with high luminosities. The plans includes guidance from the PAC and has been reviewd by DOE. The main technical improvements expected for Run- 9 are:

- Longitudinal stochastic cooling in both rings and 1 plane transverse in yellow

- The STAR DAQ1000

- A larger ToF coverage for Star

- The reinstallation of the Phenix HBD

A well though plan exists for improving machine performance over the next 5 years. The main planned upgrades for ion-ion operations are:

- Reduction in $\beta^{*}$ (from present $80 \mathrm{~cm}$ to $50 \mathrm{~cm},+60 \%$ ) 
- Lattice with reduced IBS ( $+25 \%)$

- Blue longitudinal stochastic cooling $(+15 \%)$

- Transverse stochastic cooling $(+400 \%)$

- Transverse damper, scrubbing $(+40 \%)$

- RHIC $56 \mathrm{MHz}$ SRF ( +30-50\%)

For PP the planned improvements are:

- Reduction in $\beta^{\star}$ (from $90 \mathrm{~cm}$ to $50 \mathrm{~cm},+80 \%$ )

- Nonlinear chromaticity correction $(+30 \%)$

- $9 \mathrm{MHz}$ cavity $\left(+25 \%\right.$ at $\left.\beta^{\star}=1 \mathrm{~m}\right)$

- LEBT/MEBT + Booster injection modification ( $+20 \%)$

- Eliminate triplet vibrations. Near (half) integer working point $(+40 \%)$

- $56 \mathrm{MHz}$ cavity

- Electron lens $(+100 \%$ ? )

The noted gains above are estimates and are not independent of each other in all cases.

Run- 8 has been a successful year for operation despite the funding challenges that seriously limited the length of the PP run.

The d-Au run lasted 9 weeks, the total luminosity delivered to the experiement was 437 $\mathrm{nb}^{-1}, 199$ and $238 \mathrm{nb}^{-1}$ to Star and Phenix respectively, with a time in store of $58 \%$ of calendar time. The peak luminosity recorded was $2.7 \times 10^{29} \mathrm{~cm}^{-2} \mathrm{sec}^{-1}$.

The major accomplishments in the run have been:

- Both ring were cooled down from $100 \mathrm{~K}$ to $4.5 \mathrm{~K}$ in 11 day

- Beta* was reduced to $0.7 \mathrm{~m}$ (noominally) in IP6 and IP8 in both rings

- Commission yellow lattice with higher phase advance in the arcs to reduce IBS

- Introduced periodic orbit correction at store in both rings

Aspects that still need explanation:

- Unknown noise source that sporadically appeared and cause blue transverse emittance growth

- Inconsistency of luminosity and emittance measurements

- Yellow beam lifetime of $10 \mathrm{~h}$ vs. $30 \mathrm{~h}$ in Run-7 (while colliding with another gold beam)

The goals for delivering luminosity and FOM for the PP run were met according to projections and the luminosity development was quite fast. Within only 6 weeks of physics the average store luminosity increased by only $15 \%$ compared to Run- 6 to $23 \times 10^{30} \mathrm{~cm}^{-2} \mathrm{sec}^{-1}$. The time at store was almost $60 \%$, the established goal for the facility. However, for most of the run no rotator ramps were done, which would have reduced the time in store by about $2 \%$. A configuration with $\beta^{*}$ of 0.65 (nominally) was demonstrated for future operations. However there were several challenges and not enough time (the run lasted 6 weeks) to address them effectively:

- The near integer working point did not work due to the amplified effect of triplet vibrations on the closed orbit, that produced an unacceptable beam decay and background conditions. 
- AGS polarization in the range $45-60 \%$ was lower than the $65 \%$ achieved in Run6. (half of the difference can be attributed to changes in the source, the other half to the limited AGS tuning time)

- The ramp acted as an intensity filter for the yellow beam.

Three days were dedicated to test at low energy. The $9 \mathrm{GeV}$ set-up from 2007 was successfully reproduced and improved, with a different sextupole configuration including polarity changes in some families, with the confirmation of collisions followed by a short run for physics, after solving the challenges of cogging at $h=366$, finding Phenix collisions and setting up the experiments triggers. The 56 bunch stores had much better injection efficiency and lifetime than in 2007. Vernier scans confirmed an average luminosity of $1.2 \times 10^{23}$ and maximum of $3.5 \times 10^{23} \mathrm{~cm}^{-2} \mathrm{sec}^{-1}$. The exploration of operations at $5 \mathrm{GeV}$ was much more difficult: the blue ring was unavailable due to the failure of b4-dh0, and the yellow ring proved to be a rather nonlinear machine at the energy due to large lattice sextupole errors.

For STAR the d-Au run was a success: the minbias and high-tower trigger luminosity goals were exceeded, $80 \%$ of the FMS original and $160 \%$ of the revised goal were achieved. The polarized proton run harvested good comparison data for FMS and GGC, adequate on single spin asymmetries although short of the goal, not enough data on direct photon asymmetries. The low energy run was very successful with the confirmation of real collisions and a luminosity of $1-310^{23} \mathrm{~cm}^{-1} \mathrm{sec}^{-1}$, corresponding to $\sim 0.9 \mathrm{~Hz}$ rates. The test of the newly installed PP2PP went well with measured rates as expected and no impact on Star and Phenix backgrounds. The main new developments for Run-9 are the completed DAQ-1000 and an improved ToF system. As always, we received from STAR frank and useful input on operations issues that were eventually discussed at the Retreat, and will hopefully trigger improvements especially in the areas of communication, planning and meeting management.

For Phenix too the d-Au run was very successful with a total of $80 \mathrm{nb}^{-1}$ recorded luminosity, with peaks of $3 \mathrm{nb}^{-1} /$ day. RHIC delivered more luminosity in one day of Run8 than the entire Run-3. The focus of the PP run for Phenix was development of luminosity and polarization, and on this front the collaboration expressed concern about the ripercussions of Run- 8 on the future of the spin physics program. Other issues were raised that provided good input for the Retreat and were addressed in the sessions:

- Control of backgrounds and in particular the effect of auto-orbit corrections

- Prompt analysis and results from Vernier scans

- Fast and more reliable polarization measurements

- User friendliness and reliability of the Phenix new IRIS scanner

The APEX program ran in Run- 8 with an availability of $83.4 \%$ and a scheduled/planned time of $97 \%$, in line with last few years. We ran 13 APEX sessions in Run-8: data and details can be found in http://www.c-ad.bnl.gov/APEX/APEX2008.

We had very good results this year, including the immediate payoff on luminosity following the $\beta^{*}$ squeeze to 0.7 in $\mathrm{d}-\mathrm{Au}$ and the demonstration of $\beta^{*}=0.65 \mathrm{~m}$ for future PP 
operations. Following is a summary of the experiments performed in Run- 8 with their associate relevance.

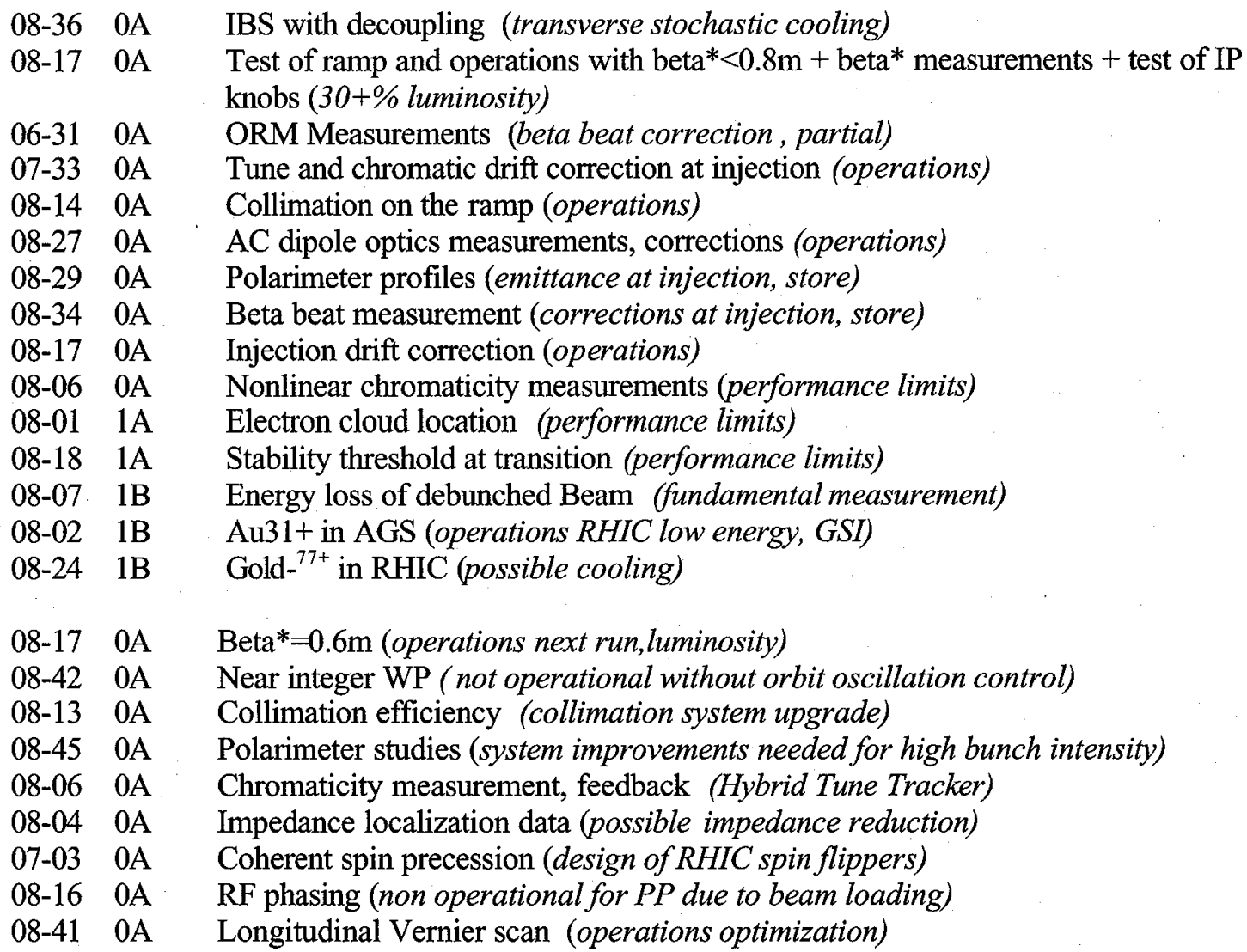

Among the goals for the APEX program in Run-9: convincing linear optics corrections, further development of IBS lattices, spin experiments, long range beam-beam compensation, chromaticity feedback and instrumentation developments.

\section{Preparation for gold-gold operations in Run-9}

The goals for the next Au-Au run have been clearly defined in the projections for Run9, http://www.cadops.bnl.gov/RHIC/Runs/RhicProjections.pdf. In particular, the projected luminosity per week is $610 \mu \mathrm{b}^{-1}$. This can be achieved with 103 bunches, $1.1 \times 10^{9}$ ions/bunch, a $\beta^{*}$ of $0.7 \mathrm{~m}$, and at $60 \%$ time in store per week. The peak luminosity is projected to be $39 \times 10^{26} \mathrm{~cm}^{-2} \mathrm{~s}^{-1}$ and the store average luminosity of $17 \times 10^{26} \mathrm{~cm}^{-2} \mathrm{~s}^{-1}$. These are achievable goals, on the basis of past runs performance.

The best Au-Au performance was in Run7, where we ran operations with 103 bunches, $1.1 \times 10^{9}$ ions/bunch and a $\beta^{*}$ of $0.85 \mathrm{~m}$. The peak luminosity was $30 \times 10^{26} \mathrm{~cm}^{-2} \mathrm{~s}^{-1}$; the store average luminosity of $12 \times 10^{26} \mathrm{~cm}^{-2} \mathrm{~s}^{-1}$, and the weekly luminosity reached $380 \mu \mathrm{b}^{-1}$ 
per week. Time at store was only $48 \%$. That was addressed at the 2007 Retreat, worked on in the ensuing shutdown and availability increased to almost $60 \%$ in Run- 8 .

The main focus in the next Au-Au run will be the operational use of the Blue longitudinal stochastic cooling system in addition to the upgraded Yellow longitudinal system, and the test of transverse stochastic cooling in the Yellow vertical plane. Experimental evidence shows that the beam intensity is limited by fast transverse instabilities at transition, driven by the machine impedance and electron clouds. So there will be a significant focus on the transition crossing and efficiency. In addition, we will run an IBS lattice in both rings, possibly with higher phase advance.

The production and transport of the beams through the injectors will remain the same as in the last run, with some minor changes. The main area of effort is in the longitudinal emittance improvements. Results from Run- 8 show that by carefully configuring the RF parameters we can significantly reduce the longitudinal emittance. These improvements will be made operational in Run-9. In addition, it is planned to replace one RF cavity in the AGS over the shutdown. This is not a performance improvement, but it will improve the flexibility and operation of the system.

An area of concern from Run- 8 is the loss of vacuum in the AGS due to loss-induced failure of vacuum chambers. This happened three times during the run. Leif Ahrens described these failures and their most likely cause. The failures were correlated with the dumping of high intensity Au beam in areas other than the AGS beam dump. The Au beam, when one considers the amount of energy deposited from energy loss, is equivalent to a high intensity proton beam $\left(5 \times 10^{9} \mathrm{Au}\right.$ ions is equivalent to $31 \times 10^{12}$ protons). Over the shutdown the beam dump will be moved closer to the circulating beam, to allow it to better intercept the beam. The optimization of the beam dump will be given priority during the Run- 9 beam setup period in AGS, and improved monitoring, alarms, and logging will allow us to better monitor and diagnose beam losses.

In Run-8, the Yellow longitudinal stochastic cooling system was used again operationally and the Blue low level completed (with the microwave link). By using the microwave link the signal arrives $200 \mathrm{nsec}$ ahead of the beam. $80 \mathrm{nsec}$ are needed to fill the kickers. With all the other delays in the system the kick to the beam occurs just in time. The desired phase margin was achieved and the system is stabilized with a pilot tone, which allowed the signal to be stable in within a few psec. A number of open issues will be addressed over the summer shutdown period. One of these are electric feed-through with vacuum problems. New ones will be built in house. For the transverse cooling system the closed orbit rejection of the transverse pickup needs to be improved. There is low power in the revolution harmonic line at $6 \mathrm{GHz}$, but the power in this line becomes very large at $5.5 \mathrm{GHz}$. Appropriate filters have been designed, but it would also help significantly if the accelerator working points were close to the half integer. The long range plans and projections for luminosity increases were addressed. The main effort for the longitudinal cooling will be using microwave links for both Blue and Yellow rings. Although the plan is to install the microwave link for Yellow this shutdown, it won't be possible to move the Yellow pickup and kicker until next year. Then the two-turn filter used in Yellow can 
be removed and it will be possible to cool the core of the beam. Transverse cooling requires some new technology (IIR filter), but the main new aspect to the system will be the interaction of transverse and longitudinal cooling. Another new aspect is the effect of betatron coupling between horizontal and vertical during cooling and the need to assess if we need both vertical and horizontal cooling systems. Cooling using a strongly coupled lattice may work. Installing two transverse pickups per ring will open the possibility of operating at any betatron tune. For now the tune will have to fall within a small fixed range of values and that limits operational flexibility. A fact we need to be aware of when running with cooling on is that the bunches will become shorter, and that this will increase the IBS emittance growth rate. The transverse cooling system will go into Yellow for the next run and the results with beam with simultaneous transverse and longitudinal cooling will guide the system future evolution.

In longitudinal transition crossing, two significant problems are the large longitudinal oscillations after transition that needs damping, and the control of the radius of the beam during transition crossing. The quadrupole feedback system that was successfully tested in Yellow in Run-8 resulting in a 10\% longitudinal emittance reduction.

This system was operational for last two weeks of the d-Au run. For Run9 a Blue damper system and upgrade from a test board into the new RF LL system will be implemented.

A dipole radius feedback system has been developed for radial control during transition. The difference in field calibration between the two rings is corrected to minimize the frequency error in Yellow (since Yellow is slave to Blue). In Run8 the loop was successfully closed. There is a time delay that needs to be understood but the expectation is to be ready to fully test the system in the next run. Another way to relax on the frequency error tolerance is to have the transition jumps occur in the two rings at different times. This is being investigated, but is complicated by the additional constraint imposed by the IBS suppression lattice. To reduce sensitivity to instabilities during transition the Landau cavities could be fixed to operate correctly at transition. This requires changing the harmonic of the cavities from 2563 to 2580 (to give a phase shift from one bunch to the next of 180 degrees). This means the cavities need to be stretched by about $10 \mathrm{~mm}$. Whether this will be ready for the next run, depends on the length of the shutdown and on bench test results with the spare cavity. Another effort to reduce beam loss during transition crossing $i \mathrm{~s}$ to lower the $\mathrm{RF}$ voltage during transition. This will lengthen the bunches and reduce the electron cloud density and thereby also the instability threshold.

All ions cross transition at about gamma 23 (26 for the IBS suppression lattice used in Run-8). The transition jump changes the gamma of the transition point by about 1.0 . Below transition the chromaticities need to be negative while above they need to be positive. At some point the chromaticities cross zero and when this happens it can cause losses. There are a few possible cures.. One possibility is to use a fast chromaticity change (using existing power supplies). Another method is to use octupoles. The preferred method is to implement a fast chromaticity jump. Data from button BPMs show a head ail oscillation and a transverse instability that persists for $50 \mathrm{msec}$. Data also indicated that the octupoles did not improve the ramp efficiency above a certain strength when looking over many stores. A study of the chromaticity jump was done at injection, 
by changing the gamma quadrupole polarities. Setting the sextupoles differently can control the resulting jump, and that was in good agreement with the model.

The performance of the IBS lattice has been analyzed. The normal RHIC FODO lattice has a phase advance of 82 degrees per cell. In Run- 8 we ran the Yellow ring using a lattice with a phase advance of 92 degrees per cell. Based on Run-7 measurements during tests of the 92 degree lattice, the transverse IBS growth rate was suppressed by $\sim 30+/-$ $10 \%$. The actual performance comparison of the IBS and the regular lattice in operations could not be entirely done for lack of clearly comparable data (see below). The expected advantages are a smaller emittance growth, and larger bucket area. These have the potential of improving luminosity lifetime and average luminosity. Other advantages are the lattice allows for lower beta-star due to slower emittance growth (when the dynamic aperture shrinks) and more relaxed power supply currents in the beta-squeeze. The main concerns are the longer set-up time to develop a more aggressive IBS suppression lattices, the higher main quad current (a potential issue for reliability), and the nonlinear characteristics of the lattice (change in dynamic aperture, and possible effects on beam lifetime). The main limit in dynamic aperture at store comes from the IR triplets and not from the arcs. Simulations are however planned to verify and further probe the impact of the new lattices.

Run-3 and Run-8, both d-Au, where compared in the attempt to correlate similar conditions and machine states. There are a number of differences between the two runs though (Run-3, no stochastic cooling, no NEG coated pipes, number of collision points, etc.). For all the data analyzed, luminosity lfetime, emittance growth $/ \mathrm{hr}$, and Yellow beam lifetime vs. bunch density, the data points fall on the same curves. In Run- 8 the normalized peak luminosity increased, when he compared the luminosity vs. fill numbers for the five best stores, for various conditions. Looking at Yellow lifetime, in the same way, it is clear that the Run-8 lifetime was greatly reduced (from $30 \mathrm{~h}$ to $10 \mathrm{~h}$, with longitudinal stochastic cooling in both cases) in comparison to Run-7, although what to properly attribute that reduction to is unclear. What is clear is that more simulations need to be performed to better understand the impact of the stochastic cooling, changes in dynamic aperture due to the IBS suppression lattice, and other factors, such as the beambeam effect with a beam that is growing less transversely and changes in beta-star. Power supply statistics were compared with the conclusion that no increase in failure failures can be attributed to the new lattice. The conclusion is that the IBS lattice should be used in operations for both rings and be studied further in APEX.

Concerning low $\beta^{*}$ developments, in the $d-A u$ run $\beta^{*}$ was first reduced in yellow and then in blue by $\sim 20 \%$ with an overall increase in luminosity of $\sim 20 \%$. In the $100 \mathrm{GeV}$ PP run we nearly commissioned $100 \mathrm{GeV}$ pp the lattice beta* of $0.65 \mathrm{~m}$ (nominally). This will continue in Run-9. At $100 \mathrm{GeV}$ this is equivalent to a beta-star of $0.4 \mathrm{~m}$ at $250 \mathrm{GeV}$ (the present power supplies can't do that). The ramp efficiencies were good: $93-95 \%$ in both rings. Correction of nonlinear chromaticity at store and correct interpolation on the ramp are mandatory. Backgrounds do go up, so collimation is equally important, as well as correction of nonlinear effects in the triplets. Open issues to test in operations are luminosity lifetime (how much worse do they become?), $10 \mathrm{~Hz}$ generated by low- $\beta$ triplets (orbit and collimation reproducibility), dispersion, and optics. About physical 
aperture in the triplets, the conclusion was that it is not a major concern. For 12 sigma full aperture, $\beta^{*}=0.65 \mathrm{~m}$ is achievable for $40 \mu \mathrm{m} 100 \mathrm{GeV}$ Au beams. Collimation really does improve background/ZDC rates based on studies performed in Run-8. Nonlinear chromaticity corrections worked well. For dispersion at the IR's, when pushing beta*, the dispersion suppression is the first thing to go (i.e. it becomes non-zero at IR) and it was expected that this would worsen with crossing angles and DX nonlinearities in the d-Au lattice. The Blue ring after nonlinear chromaticity correction showed excellent dispersion match. The Yellow ring without nonlinear chromaticity correction wdid not. This correlates to lower yellow beam lifetime and diminished yellow momentum aperture.

The dispersion telescope power supply constrains the achievable beta-star. A push to $0.4 \mathrm{~m}$ with $\mathrm{Q} 10$ shunts, larger feed-throughs and power supplies for Q89, is achievable, but the Q10 shunts are expensive. A detailed study on lower beta* options is recommended.

Following is a discussion of transverse emittance measurements and techniques. Although the discussion was mostly focused on polarized proton emittance measurements, it is useful to review here, since many issues are common between ions and protons. After listing all the devices in RHIC and the RHIC injectors for measuring emittance, the conclusion is that the overall picture is consistent, even though there are issues with the different emittance measuring devices. Emittance is a key parameter for luminosity, and it is also correlated with polarization. The P-CNI polarimeter can be used as a wire scanner and the emittance in AGS can be measured using the IPM. The results are reproducible. The relative comparison between the AGS IPM and CNI scans show the same trend and dependence on intensity, while the absolute measurements need to be better understood. The CNI polarimeter can give very nice bunch-by-bunch emittance measurements. There are other measurement techniques being developed, a residual gas luminescence monitor from the hydrogen jet target and the ratio of the power in the HF Schottky lines as a function of the device position. Looking at the emittance evolution in the entire accelerator chain, there is clearly an (understood) emittance growth in the LINAC, ending up with about 8 to $10 \mu \mathrm{m}$ beam size. There is also a few $\pi$ $\mu \mathrm{m}$ more emittance growth due to the Coulomb scattering in the injection foil. Our knowledge of the emittance in the Booster is not satisfactory, mostly due to discrepancies between measurements in the BtA and what is expected from the LINAC emittance and projected growth from the foil scattering. Plans for next year include more work on the RHIC IPMs, upgrades of the CNI polarimeters and upgrades to the Schottky systems.

In the area of longitudinal emittance preservation, by observing the evolution of the emittance from Booster injection through to RHIC for $\mathrm{Au}$ ions, it can be clearly seen that there is a very large emittance growth through the accelerator chain up to beams at store in RHIC. At Booster injection the emittance is $0.022 \mathrm{eV} \mathrm{sec/nucleon.} \mathrm{At} \mathrm{RHIC} \mathrm{store} \mathrm{it}$ has increased to $0.8 \mathrm{eV} \mathrm{sec/nucleon.} \mathrm{For} \mathrm{RHIC} \mathrm{rebucketing} \mathrm{at} 100 \mathrm{GeV}$ the requirement is that the emittance be smaller than $0.6 \mathrm{eV}$ sec/nucleon. For the low energy runs it needs to be even smaller ( 0.25 to less than $0.1 \mathrm{eV}$ sec/nucleon). There is a large emittance growth (factor of 5) in AGS and another factor of two on the RHIC ramp.This is how emittance growth was reduced in AGS: a bad place for emittance growth is during the bunch merging gymnastics, to take the 24 at AGS injection from four Booster fills to four 
bunches for transfer to RHIC. This is done in two stages. First there is a merge of two bunches to one, to get to 12 , and then a second merge of three to one, to get to four. For the second merge the RF voltages were wrong. Re-calibrating the $L 10$ cavity $(h=8, h=4)$ voltages and fixing voltage profiles, basically eliminates the emittance growth at the 3->1 merge. Moreover, by looking carefully at these merges and the match between the Booster and AGS, $0.1 \mathrm{eV} \mathrm{sec/nucleon}$ is achievable at injection. An important conclusion here is that there is no need for low energy electron cooling in AGS to meet RHIC requirements. But, the emittance still grows in AGS. The phase of the merges were also found off by 3 degrees. This should be easy to fix when setting up the merges. There is still emittance growth on the ramp and this appears to be correlated to magnetic field ripple with a resonance at the synchrotron frequency of $1440 \mathrm{~Hz}(24 \mathrm{x} 60 \mathrm{~Hz})$. This will be reduced next year after the replacement of the AGS main magnet transformers, which will reduce the ripple during acceleration. The emittance growth in RHIC grows from 0.3-0.4 to 0.6-0.7 during acceleration (steadily) and at transition. This has yet to be understood fully. A number of action items resulted from this analysis: In the AGS a new RF cavity is being installed, replacing L10, but we still need to do the merge right. The emittance growth on the ramp is not fully understood, although it looks like a major contributor is a resonance between ripple and synchrotron frequency. In RHIC, optimization a transition, including the quadrupole mode damper is very important. Finally more studies and simulations need to be done to understand the emittance (steady) growth on ramp, and longitudinal dampers should be considered for AGS.

\section{Action items for Ion run preparation.}

- Stochastic cooling is no longer a parasitic effort. Stochastic cooling plans be part of the daily schedule.

- Transition: make quadrupole feedback, radial feedback and counter-phasing operational in Run-9.

- Transition: fully understand and set chromaticity jump at transition. Formation of a transition working group.

- Change in harmonic for Landau cavities? Depends on RF group work schedule and shutdown length.

- IBS lattice: develop and determine the optimal lattice with high(er) phase advance in the arcs for Run-9 (both rings), with the additional constraint of having the gamma transition jumps at different times for each ring.

- Beta*squeeze: need to determine the optimal beta* for operations $(0.7 \mathrm{~m})$. Prepare:

1. Optics corrections

2. Nonlinear chromaticity corrections

3. IR corrections, sextupole, octupole, dodecapole

4. Collimation (on the ramp and at store)

- Continue improvement in transverse and longitudinal emittance control.

- Continue to focus on getting correct and consistent emittance measurements.

- Physics will not to be declared until Vernier scans have been completed and luminosities and cross sections are understood. Does this need additional support or efforts? (Faster time to get an answer?).

- Improved monitoring, alarms, and logging of AGS beam losses 


\section{Preparation for polarized proton operations in Run-9}

While the luminosity during Run-8 reached similar levels as in Run-6, beam polarization was significantly lower. This can be partially attributed to the short duration of the run, which did not allow sufficient time to scan various parameters, such as tunes and orbits, to improve the situation. The most likely candidate for poor polarization efficiency on the Yellow RHIC ramp has been identified as the horizontal orbits at the two snakes not being parallel. Correction of this was hampered by the unavailability of two BPMs in the vicinity of one of the Yellow snakes: these BPMs will be repaired in preparation for Run9.

In the AGS, the best polarization at extraction into RHIC reached only $60 \%$ at an intensity of $1.510^{11}$ protons/bunch, compared to $65 \%$ in Run-6. At AGS injection, the polarization was about 4 percent lower than in Run-6; furthermore, a significantly steeper polarization profile was observed. More realistic simulation studies will be carried out, including sextupoles and orbit bumps, to gain a better understanding of polarization in the AGS. The planned RHIC low-level RF upgrade will possibly facilitate extraction on the fly out of the AGS. This will increase the ramping speed across the last depolarizing resonance in the AGS at $36+v y$ which is expected to decrease the polarization loss at this resonance. Higher injection energy into the AGS is expected to result in less emittance growth at injection energy. Injection on the fly and higher injection energy in AGS will not likely be operational in Run-9. A dedicated set of quadrupoles will be installed to enable tune jumps at all weak horizontal spin resonances. This system will be operational in Run-9.

The polarization issue in Run- 8 was somewhat confused by different analyzing powers between the AGS and RHIC polarimeters, and by discrepancies between RHIC injection and store, especially in the Yellow ring. These issues need to be resolved by crosscalibration of the various polarimeters. For this purpose, it is planned to run the JET polarimeter at RHIC injection energy for about 20 hours in order to provide calibration data for the CNI polarimeter at this energy.

The CNI polarimeters will be upgraded during the summer shutdown. The Yellow polarimeter will be outfitted with the same new motor drives as in Blue. Furthermore, two identical polarimeter vessels will be added, duplicating the existing CNI polarimeters. This will allow for simultaneous polarization scans in both the horizontal and the vertical plane. Testing of equipment such as new detectors will also be enabled in this configuration, by installing the equipment to be tested in one of the polarimeters, while leaving the other one unchanged. This is especially important due to potential limitations of the existing CNI polarimeters at high intensities, which requires new hardware. 
At the end of Run- 8 a new two-beam mode at the JET polarimeter was tested successfully. Both beams were simultaneously brought into the acceptance range of the JET detectors; the beam separation at the JET was lowered to $5.5 \mathrm{~mm}$ for this purpose. This new mode facilitates measuring the polarization of both beams simultaneously, which effectively doubles the amount of data taken. Furthermore, the reed for frequent orbit swaps at the JET will be eliminated, thus contributing to more stable operation of the facility.

During several APEX and Machine Development sessions in Run-8 a $\beta^{*}$ squeeze to 0.65 $\mathrm{m}$ was successfully attempted. Up to 56 bunches were accelerated and brought into collision. After inserting the collimators, background levels comparable to regular $1 \mathrm{~m} \beta^{*}$ were achieved. Attaining good beam lifetimes at store requires nonlinear chromaticity correction, which was successfully demonstrated as well.

A new $9 \mathrm{MHz}$ RF cavity for RHIC polarized protons acceleration will be installed during the summer shutdown. This new system is expected to reduce the longitudinal emittance at store by a factor two; the resulting peak current reduction may also help in preventing transverse emittance growth on the ramp. The bunch length reduction will reduce the hourglass effect, which will be a significant improvement especially at the reduced beta*. Furthermore, the shorter bunch length will reduce the vertex distribution at the detectors. Sufficient commissioning time needs to be scheduled at the beginning of the polarized proton run in Run-9 to make this system operational. If protons will be run during the initial part of Run-9, the low-level RF upgrade requires dedicated commissioning time as well. A comparison of effective ZDC cross sections over several runs has revealed large year-to-year fluctuations of these parameters. For instance, effective cross sections at both STAR and PHENIX were measured at roughly 0.3 mbarn in Run- 8 , vs. approximately 0.5 mbarn in Run-6. The Run- 8 value is in good agreement with runs before 2003. Possible reasons for the year-to-year changes are different set points for the high voltage at the ZDCs, other electronics changes, or added shielding. A known difference between Run- 6 and Run- 8 is the lack of correction for both singles rates and the hourglass effect in determining the effective cross section in Run-6. In Run-9, a faster, online analysis of Vernier scans will be operational that will provide effective cross sections early in the run.

In Run-9, an average store luminosity of $4010^{30} \mathrm{~cm}^{-2} \mathrm{sec}^{-1}$ should be attainable due to the tighter beta* squeeze to $0.68 \mathrm{~m}$ the $9 \mathrm{MHz}$ cavity, and nonlinear chromaticity correction at store. This goal is the same as for Run-8, which was too short to reach it.

\section{Machine Operations and Systems}

The time at store for Run- 8 was $58 \%$ for $\mathrm{d}-\mathrm{Au}$ and almost $60 \%$ for P-P operations respectively. $60 \%$ is the goal that we established for the facility and represents a welcome trend inversion, after 2 years of operations (Run- 6 and 7) where the uptime dipped under $50 \%$. The focus on machine reliability at the last Retreat and during the shutdown paid off. The availability - as defined in the DOE metrics - also was above the goal of $80 \%$. 
In this Retreat we examined again uptime and system reliability to discuss new challenges, improve on old ones and see where we can in general do better.

In Run- 8 we had overall 516 failure hours (16\% of time) or 3.8 hours/day be compared with the Run-7 overall 881 failure hours $(28.6 \%$ ) or 6.9 failure hours/day, a clear progress. The distribution of failure as well as the comparison with previous run is summarized in the following table:

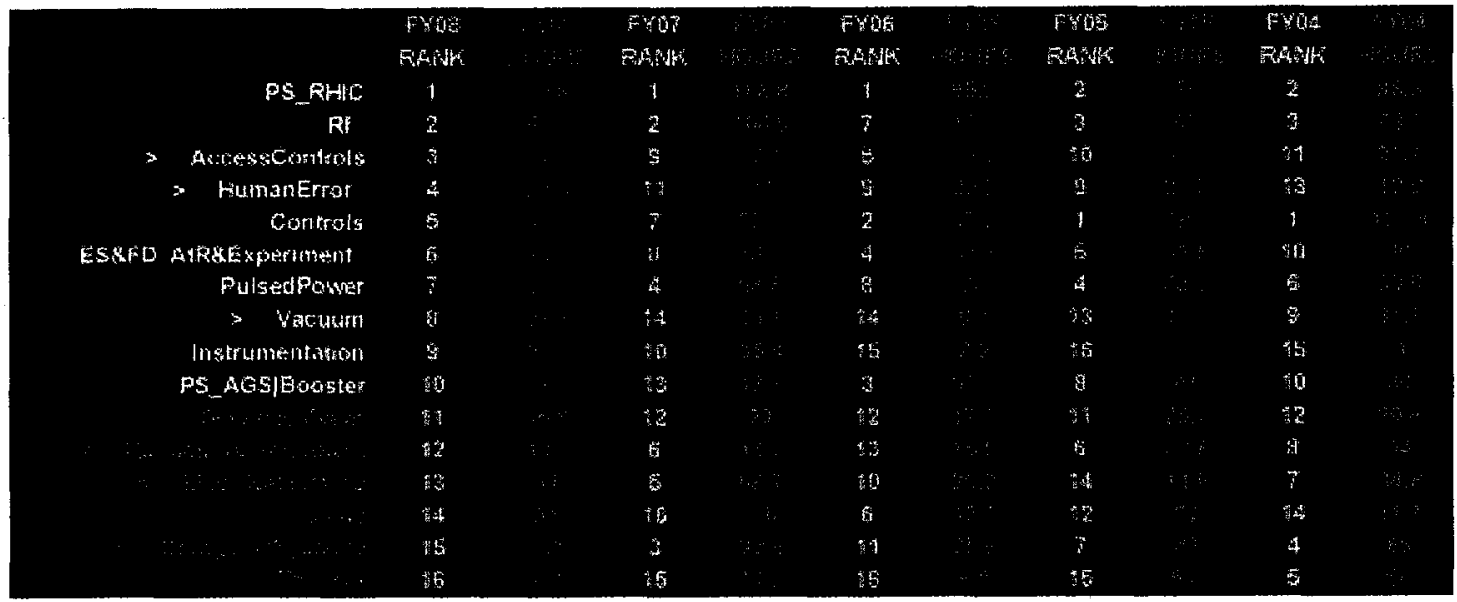

While RHIC power suppies still account for most of lost hours, the failure time was greatly reduced from last year, as the efforts on increasing PS reliability, particularly the IR PS, paid off. RF system reliability improved noticeably, as well the pulsed power systems and instrumentation. ACS and vacuum system failure increased, the latter partly caused by the AGS vacuum problems generated by the internal dump. Another visible increase has been in the human error category, which motivated further analysis.

Human error events have been analyzed in terms of human performance, to identify the most recurrent and to have a guidance where to focus improvement. Almost $60 \%$ are instances of "rule based" failure model which is the misinterpretation of rules. "Skill based" errors (inattention during familiar and routine situation) account for $30 \%$ and only $10 \%$ fall in the "knowledge based" category, which reflects an inaccurate mental model of a process in an unfamiliar situation. An analysis of 597 fills during Run-8 d$\mathrm{Au}$ operations established that 13 fill terminations could be attributed to operations personnel (operators, OC), most frequently caused by miscommunication, and 24 terminations to system expert and physicists, mostly caused by system configuartion errors. Operations will work in the following areas to reduce the likelihood of human errors:

- Peer and self checking, independent verification

- Prejob briefing

- Procedure imrovement, use and adherence

- Improved alarming, software safeguards

- Questioning attitude and situational awareness 
An essential factor in efficient operations is the organization of maintenance and repairs. The methods outlined at the 2006 Retreat, namely:

- Pre-set access time

- Approval process

- Ownership- Affected group re-affirms that systems are operational before jobs are completed

- Closeout Statements- Operational test results, owner accord and future testing improvements

- Documentation

- Post mortem analysis

- WEB based Job Request System

have been systematically applied and extended resulting in clear improvements: during Run- 8 the injectors were often operational on or before the predetermined time, the impact of sweeps was minimal, a test period for injectors after access minimized overall delays. The additional CAS personnel made available during recovery periods proved very useful. The great success this year is that the average elapsed time from the beginning of a scheduled maintenance to actual RHIC physics running was brought down to $\sim 14 \mathrm{~h}$, compared to $22 \mathrm{~h}$ in Run- 7 and $25 \mathrm{~h}$ in Run- 6 . That was the result of allowing less unscheduled and emergent jobs, better overall access plans and especially an improvement in testing procedures. The scheduled maintenance methodology and software are being used for the management of the summer shutdown and the latter will offer the opportunity of improvement and integration of procedures and applications.

The following prioritized "wish list" is the result of discussion and polls in the operations group:

- Straightforward polarimetry

- RHICInjection without idiosyncrasies

- A RampManager and model that won't foil tape, Lisa, OrbitDisplay, RampEditor

- PASS that works faster and with less failure

- Our fingers on the keyboard

- A fully revertible machine

- Labview applications that run and dump themselves

- One answer from one source

- A list of exceptions smaller than the list of rules

Operations identified also a set of issues that seems recurrent at Retreats where a followup is necessary:

- Vital information missing or muddled in the elog

- Keep automatic entries together

- Threaded views

- Conflicting instruction sources

- Communication with experiments

- Background targets vary from shift to shift

- Phone calls are important but need a (logged) backup

- Injection tuning

- Still many bugs, slow or unresponsive

- PASS 
- MCR oversight of APEX, Development periods

- Sequencing

Pressure increases in AGS brought unexpectedly down the injectors in Au operation while using the AGS internal J10 dump. Pipe damage was eventually confirmed. While the radiation generated by $5 \times 10^{9} \mathrm{Au}$ ions at $10 \mathrm{GeV}$ is small (equivalent to $\sim 4 \times 10^{11}$ protons at $25 \mathrm{GeV})$ the heating of the vacuum pipe by the beam is substantial $\left(\sim 3 \times 10^{13}\right.$ protons at $25 \mathrm{GeV}$ ). A thorough analysis of the internal dump was conducted leading to a proposed overall improvement strategy for the dump, including moving the dump closer to the circulating beam, increasing performance of the J10 dump bump PS, and further strengthening monitoring, logging and alarming for the bump.

RHIC polarimeter operations has been a problem during Run-8. On the one hand, the polarimeter has by design many operation modes and configurations for different measurements:

- Polarization of the beam center

- Polarization profile of the beam (Vertical and Horizontal)

- Beam intensity profile

- Beam emittance by bunch

- Polarization at injection

- Polarization on the ramp

The consensus in operations is that the instructions were complex, and constantly evolving. Moreover, dynamically changing software contributed to the problem by slowing down response and analysis. One suggestion could be offering polarization profiles as the default operation mode and handle all other functionality as an expert system. Whatever the adopted solution may be, it is clear that operations needs an agreed upon proposal from the polarimeter group well ahead of the next start of PP operations.

Vibration if the triplets in frequencies around $10 \mathrm{~Hz}$ have been recognized as a source of orbit jitter in operations. While tolerable at the usual tunes, they prevented the use of the planned new working point near the integer in PP operations in Run- 8 because the amplification of the closed orbit variation made the machine inoperable. Future developments require compensation or corrections of the vibration. Analysis and experiments allowed to establish the following conclusions:

- The triplet magnet and cryogenic line supports are unique (different from the arc magnets)

- There is no direct way to measure magnet motion at this time.

- A connection between magnet line flow rate and triplet induced beam oscillations has been established.

- Flow in the recooler supply and return lines does not have an effect on vibration.

- Thermal acoustic oscillations from the leads has been tested and found not to affect the vibration

An engineering analysis is in progress to determine the most technically sound and cost effective way to proceed. The options on the table are the following, with a rough cost estimate:

- Quad to Quad stiffening may be cost effective (IIW)

$\$ 350 \mathrm{~K}+1.5 \mathrm{MY}$ C-AD labor 
- Decoupling the magnet line may be cost effective (IIW)

$$
\$ 400 \mathrm{~K}+1.5 \mathrm{MY} \mathrm{C}-\mathrm{AD} \text { labor }
$$

- Passive stiffening expensive to implement, high heat load, possible magnet misalignment $\$ 4.2 \mathrm{M}+4 \mathrm{MY} \mathrm{C}-\mathrm{AD}$ labor

- Rebuild triplets with improved base design. $\$ 3.5 \mathrm{M}+6 \mathrm{MY} \mathrm{C}-\mathrm{AD}$ labor

- Active motion compensation - small motion compensation and sensor and base support design to be defined.

$\$ 350 \mathrm{~K}+$ labor for R\&D system

$\$ 1.0 \mathrm{M}-\$ 5.0 \mathrm{M}+2-6 \mathrm{MY} \mathrm{C}-\mathrm{AD}$ labor to implement

The plan for the 2008 shutdown is to set-up a direct motion measurement system in one triplet, to decouple the magnet line from the dipole magnet (for one triplet) and to see if it is possible to tie the quads to move in synch (again, only in 1 triplet). Future plans and choices depend of course on results, budget and schedule.

As already mentioned, although the RHIC PS retain the pole position in system failure, the progress in Run- 8 has been impressive by reducing the total PS failure hours by more than a factor of 2 w.r.to Run-7. The effort during the 2007 shutdown paid off very well: all major PS systems were improved: all PS in service building, in particular bipolar Suncraft 150A and 300A, Dynapowers. Work has been done on the QPA assemblies, the Main PS and AtR. Adding 1 more PS engineer also contributed to the group capability and effectiveness. A number of problems areas have been identified and there is an extensive list of work planned for the shutdow:

- Complete Phase 1 and 2B of SCE 150/300 mods

- Finish QPA work (including faulty relays)

- Inspect all Dynapower contactors (replace fans?)

- Repair/understand failure of b4-dh0

- Investigate B9 - 6000A quench switch problems

- Analyze mains setpoint card

- Complete 2 Instrumentation set-ups

- Clean quench detectors and install new fan trays

- Shunt bus problem repairs

- Teaching others in group QLI analysis

- List of programs given to controls to assist with start up and QLI Analysis

- Complete Air Conditioning to improve Environmental Conditions

- Install Link box in $12 \mathrm{~A}$ yellow first, coordinate blue install

The main improvement to the performance and reliability of the RF system is the LLRF upgrade, with Phase 1 planned for Run-9 startup. Phase 1 consists in replacing the functionality of the main RHIC LLRF crates with new system controllers, and eliminating the lock-in amplifiers. There are many areas in which the new LIRF will improve system reliability and flexibility, i.e.:

- Improve reliability by eliminating older problematic hardware

- Great improvement in diagnostic data capture and logging, in terms of both throughput and quantity, will greatly enhance our ability to monitor and troubleshoot the systems 
- Much improved and very tight integration between blue and yellow LLRF systems will facilitate more robust control of BLUE vs. YELLOW phasing: Ring to Ring Synchro, Collision Cogging, Rev Tick Phasing ...

- Improved control and monitoring of customer RF clocks, e.g. RevTick, Beam Sync Clock

- Ability to adjust/reset beam sync clock phase in real time with beam in the machine

- Eliminate "dead time" on beam sync clock at "cogging reset'

- Remote reconfiguration means vastly reduced downtime from system modifications, and reliable restoration from temporary reconfigurations (for APEX, etc.)

Although part of the system functionality will be tested before beam, it is important to realize that commissioning with beam will necessary.

The other major development in the RF system are:

- $9 \mathrm{MHz}$ cavity, motivated by the need to get low longitudinal emittance $(\sim 0.5 \mathrm{eV} \mathrm{sec})$ and long bunches $(\sim 20 \mathrm{~ns})$ on the ramp. This avoids transverse emittance blowup on the ramp caused by electron cloud. The lower longitudinal emittance at store will keep bunches shorter ( $\sim 6 \mathrm{~ns}$ at $250 \mathrm{GeV})$ with only the $28 \mathrm{MHz}$ cavities. The Plan is to have the cavity operational for Run-9.

- The split of the common cavities

- The L10 replacement

An analysis of all Run- 8 failure mode and causes for the Access Controls System established that $\sim 25 \mathrm{~h}$ of the overall $50 \mathrm{~h}$ were lost on faulty switches and corrosion. One specific event of water in the electric strike system that caused the failure of a gate reset system was responsible for over $11 \mathrm{~h}$ of downtime. A plan has been proposed to improve the ACS system reliability that includes:

- Replacing relays and sockets (2008 shutdown)

- Upgrade of all exterior weathered gates to 4GE2 standards (esimated cost: $~ 30 \mathrm{~K}$, as soon as money is allocated)

- Overall upgrade of AGS/Booster/Linac relay based ACS to a digital, PLC based ACS (also needed for the MCR Upgrade project, target date 2010)

In last few years of operation the Controls system has seen a steady and consistent improvement in overall reliability. Noticeable the reduction of hardware related failures, especially of radiation induced failures, a clear payoff from the radiation resistant hardware installed in the alcoves (most visible results in 7a 7c 9a and 9c).

Future effort in the controls system will focus on the following areas:

- RHIC ramping system software, with the goal of increased reliability and added capabilities

- Polarimeter measurements

- Injection system controls improvements: logging, applications, online model, Power supply and alarm management

- Motion control systems

- System administration, in particular cyber-security and strategies for OS upgrades

Concerning instrumentations systems: 
- Only 5 BPM boards failed during Run-8, best run ever. 2 were optoboards; 150/750 BPM boards remain for opto replacement. Feedthrough issues, however, are on the rise.

- Measurements confirmed that the new IPM design greatly reduced coupling between the signalgating grid and anode

- The abort gap monitor is not ready yet

- The bunch-by-bunch turn-by-turn monitor has been developed and turned to operations

\section{The Panel Discussion}

The discussion session was designed to enable all members of the Retreat to propose topics for discussion, beyond what arises from the presented talks. Retreat participants were invited to submit topics for discussion before and during the Retreat. 20 persons submitted thirty-four topics. A Panel of six Retreat members was assembled and organized the session by dividing the topics into categories that were roughly defined by the panel member's personal specialization. The panel comprised:

- Leif Ahrens, Injectors

- Peter Ingrassia, Operations

- Waldo MacKay, Spin

- Rob Michnoff, Controls

- Jon Sandberg, Systems (Power supply, RF, Vacuum, etc.)

- Todd Satogata, Operations Analysis.

The Panel also arranged the order in which the topics were discussed. They led the discussions by calling upon those who submitted topics, and asked the submitters to introduce the topic by showing a transparency to explain and define the subject. The session proceeded by scrolling through the categories of the Panel members and limiting the time for discussion in each topic to 10 minutes. By jumping from one category to the next between topics the discussion kept moving along without the tendency to drag one topic into the next. A wide spectrum of topics was but forth, from new notions of the role of the run coordinator to pros and cons of lattice choices. The complete recording of discussions can be found at:

http://www.c-ad.bnl.gov/RHIC/retreat2008/PanelDiscussion.htm

\section{Plans for the Shutdown}

The 2008 shutdown is in progress and these are the main planned machine developments:

- LEBT/MEBT rebuild + Booster injection change

- possibly pulsed quadrupoles in AGS

- RHIC polarimeter upgrade

- continuing work on abort gap monitor

- BPM feed through repairs 
- possibly vertical collimator upgrade

- installation of instrumentation to measure $10 \mathrm{~Hz}$ cold mass movement

- $9 \mathrm{MHz}$ cavity installation

- change in frequency of Landau cavities

- possibly move of 2 RHIC storage cavities to 3 o'clock

- replacement of L10 cavity in AGS

- RHIC low level rf upgrade

- upgrade of Yellow longitudinal stochastic cooling (microwave link installation)

- installation of Yellow transverse cooling

- AGS MMPS transformer replacement

- installation of dehumidifiers in RHIC tunnel

- installation of $\mathrm{A} / \mathrm{C}$ in RHIC service buildings

- further upgrade of RHIC IR and main PS

- repair of Blue buses (to allow higher currents)

- new file server for control system

Shutdown update and progress as well as scheduling information on the Run-9 start-up can be found on the Maintenance WEB page:

http://www.cadops.bnl.gov/AGS/Accel/Maintenance/ 